\title{
LA ACREDITACION DE CARRERAS UNIVERSITARIAS Y SU RELACION E IMPACTO EN LA CALIDAD DE ENSEÑANZA DEL DOCENTE EN EL ECUADOR
}

\author{
Armando Rene ANCHUNDIA CARRASCO ${ }^{1}$ \\ Ofelia Carmen SANTOS JIMÉNEZ ${ }^{2}$ \\ https://orcid.org/0000-0003-1294-0641
}

\begin{tabular}{|lcc|}
\hline Recibido & $:$ & 20.03 .2020 \\
Aceptado & $:$ & 15.06 .2020 \\
Publicado & $:$ & 06.07 .2020 \\
\hline
\end{tabular}

RESUMEN: este artículo de investigación trata de la una relación que existe entre la acreditación de las carreras universitarias y la calidad de la enseñanza por parte de los docentes universitos, así como también el análisis de los principales factores que puedan alimentar esta relación. El principal objetivo de esta investigación fue establecer si ambas variables, calidad de enseñanza y acreditación, tienen relación, así como de establecer los componentes fundamentales para su correlación. La investigación tiene un enfoque cuantitativo con un diseño descriptivo correlacional dado que comprende los siguientes pasos: recolección de datos de ambas variables para su descripción en función a sus dimensiones. Para tal fin se evaluará las políticas y evolución de la normatividad, así como de las estadísticas en Ecuador. Finalmente, como hipótesis se llegó a concluir que existe una correlación muy alta.

Palabras claves: acreditación, calidad de enseñanza, docencia universitaria.

\section{The accreditation of university degrees and their relationship and impact on the quality of teaching} of teachers in Ecuador

\begin{abstract}
This research article deals with the relationship between the accreditation of university degrees and the quality of teaching by university teachers, as well as the analysis of the main factors that may feed into this relationship. The main objective of this research was to establish if both variables, quality of teaching and accreditation, are related, as well as to establish the fundamental components for their correlation. The research has a quantitative approach with a correlational descriptive design since it includes the following steps: data collection of both variables for description according to their dimensions. To this end, policies and regulatory developments, as well as statistics in Ecuador, will be evaluated. Finally, as a hypothesis it was concluded that there is a very high correlation.
\end{abstract}

Keywords: accreditation, quality of teaching, university teaching.

\footnotetext{
${ }^{1}$ Universidad Nacional Mayor de San Marcos. Email: army2431@hotmail.com

${ }^{2}$ Universidad Nacional Mayor de San Marcos. Email: ofelia.santos@unmsm.edu.pe
} 


\section{Journal of the Academy $|109|$}

\section{INTRODUCCIÓN}

La educación universitaria está promovida principalmente con un objetivo, el cual es alcanzar la calidad educativa, todo ello con la finalidad de hacer frente y responder los grandes retos que la globalización impone a cada día, más aún si abordamos a la docencia universitaria que deberá adecuar nuevos paradigmas para no desfallecer y seguir siendo la principal fuente de trasmisión del conocimiento a los estudiantes.

Los nuevos paradigmas a los que nos referimos, se basan no solo en el aprendizaje de nuevos conocimientos o una constante adquisición de información, sino también el manejo de la misma a través de las diversas herramientas que los avances tecnológicos nos brindan: las TIC's de la información son ahora los nuevos desafíos para la docencia y es por la cual ahora deben parte de la vida de la enseñanza universitaria.

Sin embargo, el ser rigurosos con la docencia para su formación en estas nuevas herramientas también pasa por la evolución de las escuelas de e educación de donde provienen, es por ello que aparece la acreditación como un proceso que no sólo se trata de evaluaciones de alta rigurosidad, sino también como un proceso que asegurará la calidad de la formación de los estudiantes, las carreras y programas de estudio, que en este caso está a cargo del Consejo de Evaluación, Acreditación y Aseguramiento de la Calidad de la Educación Superior, CEAACES. El artículo 95 de la Ley Orgánica de Educación señala:

Art. 95.- Acreditación. - La Acreditación es una validación de vigencia quinquenal realizada por el Consejo de Evaluación, Acreditación y Aseguramiento de la Calidad de la Educación Superior, para certificar la calidad de las instituciones de educación superior, de una carrera o programa educativo, sobre la base de una evaluación previa.

La LOES señala que el Art. 352 de la Carta Suprema del Estado determina que el Sistema de Educación Superior estará integrado por universidades y escuelas politécnicas; institutos superiores técnicos, tecnológicos y pedagógicos; y conservatorios superiores de música y artes, debidamente acreditados y evaluados. Estas instituciones, sean públicas o particulares, no tendrán fines de lucro. Asimismo, señala:

La acreditación se concibe como una certificación de la adquisición de algo, que ordinariamente hace referencia a la calidad. Es el proceso de reconocimiento que se le otorga a una institución por parte de una organización pública o privada y, que ordinariamente va ligada al cumplimiento de estándares que pueden ser nacionales o internacionales. La acreditación habilita para el funcionamiento administrativo de una determinada institución educativa.

Asimismo, se ha considerado que en esta correlación bajo un modelo de calidad total educativa el «foco» se pone también en el «destinatario» del quehacer educativo, que es ante todo el educando, llamado «beneficiario», que ocupa el lugar del «cliente» en el ámbito empresarial. Tal posición central del «educando» es coincidente con los avances de la nueva pedagogía, que ha desplazado la importancia que en otros tiempos tuvieron los «contenidos» o los «docentes» y que ahora posee el mismo sujeto de la educación que es el educando, pero sin descuidar el rol del docente ni el de los contenidos educativos.

La investigación también ha mostrado alguna funciones y ámbitos de análisis para el proceso de evaluación con fines de acreditación, en la cual la docencia también forma parte importante para este proceso. 


\section{Journal of the Academy $|110|$}

Figura 1: Aspectos fundamentales para la acreditación

Elaboración propia con información del Reglamento del CONEA.

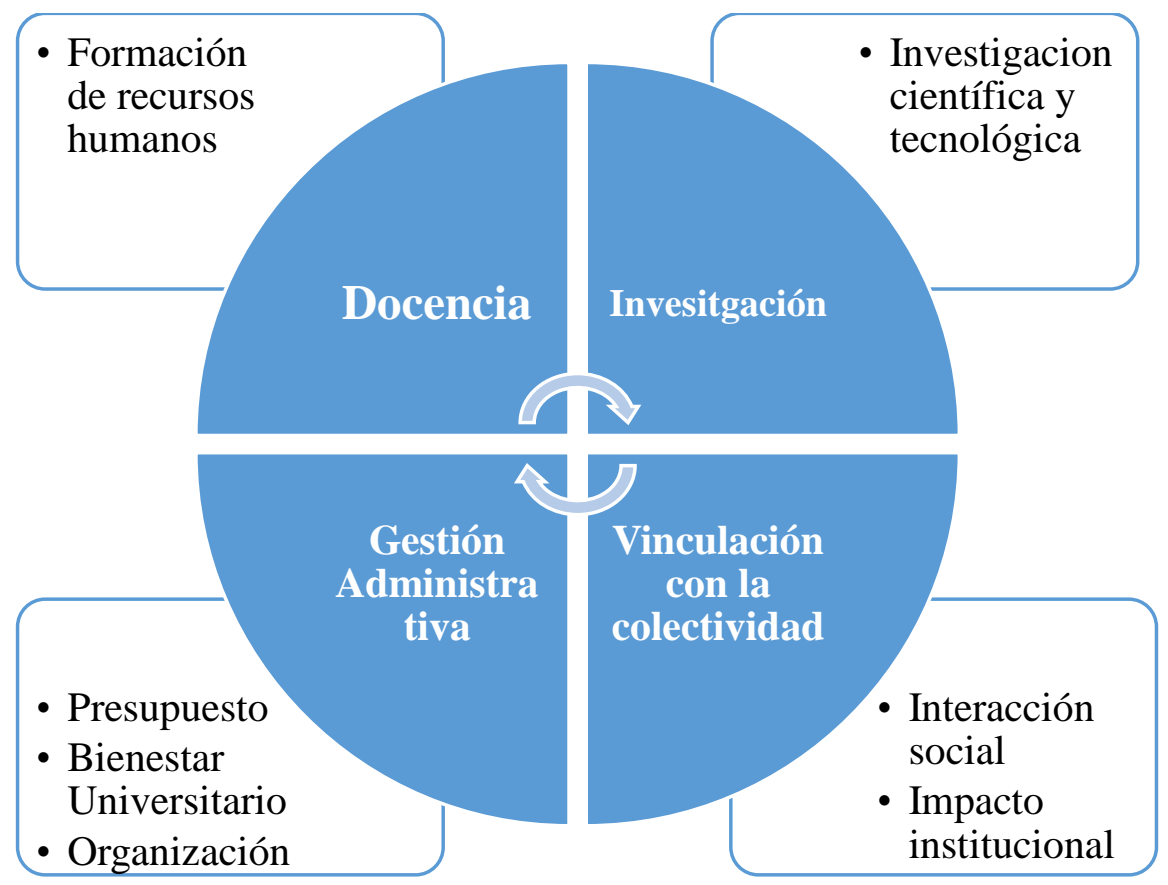

Para el caso de la enseñanza docente. Borroto y Salas (2000) proponen cómo sería el proceso de acreditación docente de una institución.

1. La autoevaluación que la misma efectúa sobre las condiciones que reúne para desarrollar un programa de pregrado o posgrado a la luz de los requisitos generales y específicos establecidos por la Universidad de conjunto con los Organismos de la Administración Central del Estado y los órganos correspondientes del Gobierno Local.

2. A continuación, la Universidad efectúa a nivel territorial la pre-acreditación correspondiente y emite su dictamen.

3. Si el dictamen es favorable, la Universidad eleva la solicitud de acreditación, con toda la documentación exigida, a la Comisión Nacional de Acreditación.

4. La Comisión Nacional designa al Grupo de Profesores de reconocido prestigio, que efectúa la verificación del cumplimiento de los requisitos establecidos. Ello conlleva además entrevistas con los estudiantes y los profesores. Y propone sus conclusiones.

5. La Comisión Nacional de Acreditación emite su dictamen y lo eleva a la consideración del Ministro de Salud Pública.

6. El Ministro o su representante, emite la decisión final.

En este sentido, con estas premisas se ha procedido a la recopilación y el análisis de la data e información estadística necesaria para la explicación de las variables.

\section{DESARROLLO}

\section{Materiales y método \\ Diseño y lugar de estudio}

Se realizó una investigación de enfoque cuantitativo basada en la recolección de datos para la probar la hipótesis planteada. Hernández, Fernández y Baptista (2006) nos dicen que "el investigador recolecta 


\section{Journal of the Academy $|111|$}

datos numéricos de los objetos, fenómenos o participantes, que estudia y analiza mediante procedimientos estadísticos" (p. 5)

Asimismo, para este caso el lugar de la investigación comprendió a la comunidad universitaria universidad Laica Eloy Alfaro, entre autoridades universitarias, docentes y estudiantes.

\section{Muestra}

López (2004) señala que es un subconjunto o parte del universo o población en que se llevará a cabo la investigación. La muestra es una parte representativa de la población. Lo cual para este caso fueron de 268.

\section{Variables}

Variable 1: Acreditación de Carreras Universitarias

Es la verificación tras la evaluación de los criterios establecidos por el Consejo Nacional de Acreditación de la Educación Superior - CEACCES la cual confirma que los programas académicos de la facultad, escuela u otro departamento académico cumple con los estándares de una educación de calidad.

Dimensiones:

- Plan Curricular

- Académico

- Ambiente Institucional

Variable 2: Calidad de Enseñanza del Docente

Guzmán (2011) señala que el concepto de calidad docente despierta debates, ya que para caracterizar a un buen profesor se debe de ir más allá de un listado de habilidades de enseñanza; la calidad es algo más que una descripción de destrezas independientes, por eso hay que tomar en cuenta otros aspectos tales como su identidad profesional, las creencias pedagógicas y su compromiso con la profesión.

Dimensiones:

- Docencia

- Investigación

- Relación con el estudiante

\section{Análisis}

En el presente estudio, los resultados obtenidos fueron analizados en el nivel descriptivo y en el nivel inferencial, según los objetivos y las hipótesis formuladas. En el nivel descriptivo, se han utilizado frecuencias y porcentajes para determinar los niveles; en el nivel inferencial, se ha hecho uso de la estadística paramétrica y como tal se ha utilizado el coeficiente de correlación Pearson ( $\mathrm{r}$ de Pearson) a un nivel de significancia del 0,05 , debido a que los datos presentan distribución normal, lo que evidencia el empleo de un estadístico paramétrico. 


\section{Resultados}

Variable: Acreditación de las Carreras Profesionales

DIMENSIÓN: Plan Curricular

\section{Cuadro 1}

\begin{tabular}{|l|l|l|l|l|l|}
\hline \multicolumn{2}{|c|}{} & Frecuencia & Porcentaje & $\begin{array}{l}\text { Porcentaje } \\
\text { válido }\end{array}$ & $\begin{array}{l}\text { Porcentaje } \\
\text { acumulado }\end{array}$ \\
\hline \multirow{4}{*}{ Válido } & Bajo & 3 & 20,0 & 20,0 & 20,0 \\
\cline { 2 - 6 } & Medio & 5 & 33,3 & 33,3 & 53,3 \\
\cline { 2 - 6 } & Alto & 7 & 46,7 & 46,7 & 100,0 \\
\cline { 2 - 6 } & Total & 15 & 100,0 & 100,0 & \\
\hline
\end{tabular}

\section{Figura 1}

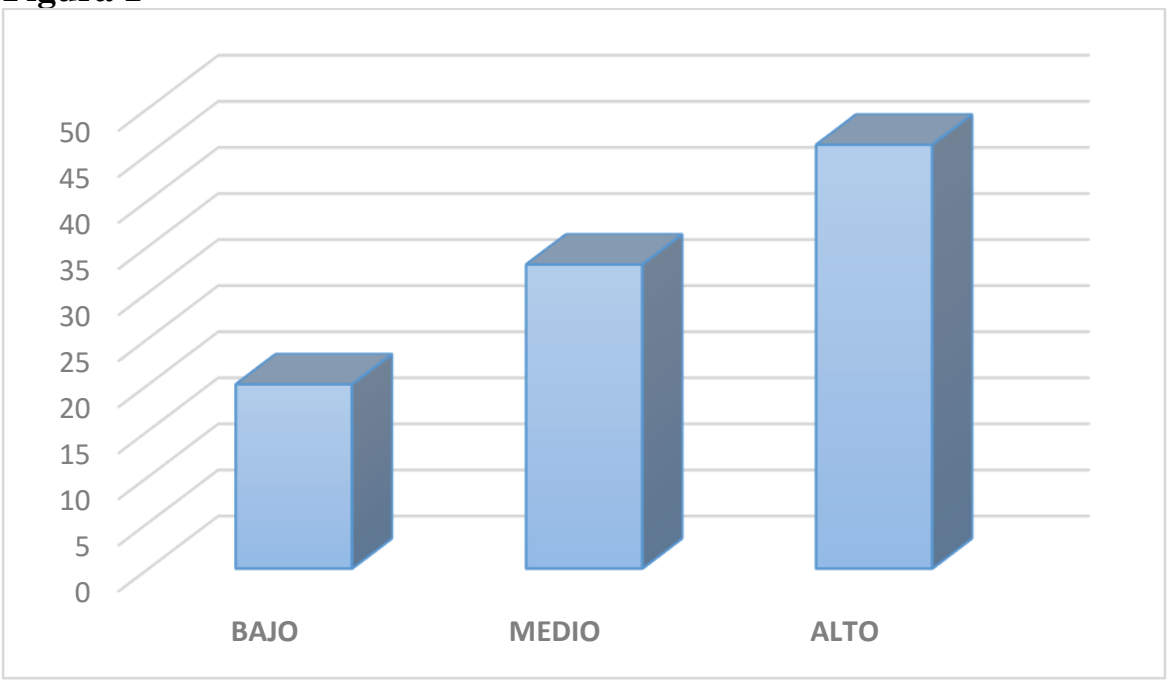

Interpretación: De acuerdo al cuadro estadístico y a la figura, se evidencia que el 46,7\% señala que es de nivel alto el plan curricular que garantiza al término de los estudios que el estudiante posea los resultados del aprendizaje estipulados en el perfil de egreso, la malla curricular indica el tipo de materia: obligatoria, optativa, práctica, la secuencia de la materia, pre requisito correquisito, el número de créditos que concede cada materia, los programas de asignaturas son completos (especifican secuencia de asignaturas, objetivos, número de créditos, contenidos, resultados de aprendizaje, mecanismos de evaluación y bibliografía), cada materia de la carrera cuenta con un sílabo o programa de la materia que especifica claramente, además de los contenidos, los resultados o logros del aprendizaje se desarrollan con los mecanismos utilizados para la evaluación, las prácticas están integradas en la estructura curricular y se relacionan con otros componentes curriculares, el 33,3\% señala que es medio y el 20,0\% señala que es de nivel bajo.

\section{DIMENSION: Académica}

\section{Cuadro 2}

\begin{tabular}{|l|l|l|l|l|l|}
\hline \multicolumn{2}{|c|}{} & Frecuencia & Porcentaje & $\begin{array}{l}\text { Porcentaje } \\
\text { válido }\end{array}$ & $\begin{array}{l}\text { Porcentaje } \\
\text { acumulado }\end{array}$ \\
\hline \multirow{3}{*}{ Válido } & Porcentaje Bajo & 4 & 26,7 & 26,7 & 26,7 \\
\cline { 2 - 6 } & Porcentaje Alto & 11 & 73,3 & 73,3 & 100,0 \\
\cline { 2 - 6 } & Total & 15 & 100,0 & 100,0 & \\
\hline
\end{tabular}




\section{Journal of the Academy | $113 \mid$}

Figura 2

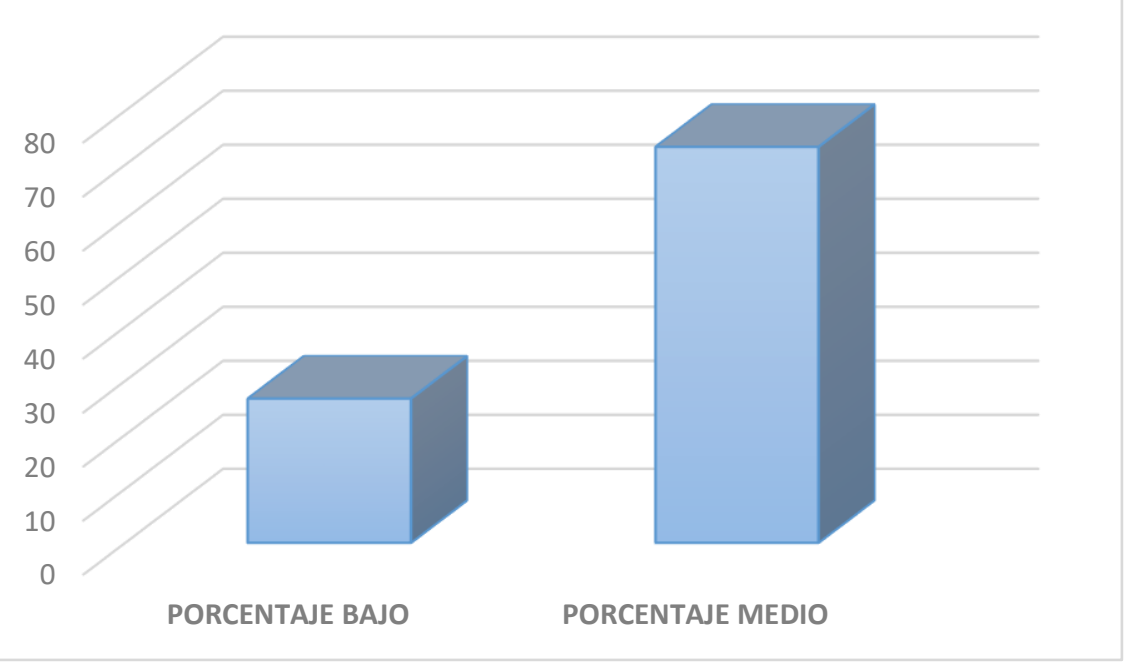

Interpretación: De acuerdo al cuadro estadístico y a la figura, se que el 73,3\% señala que el porcentaje es de nivel alto en las horas de clase realizadas por especialista, docentes con títulos de $\mathrm{PhD}$ y Maestrías, y docentes que participan en eventos de actualización, en experiencia profesional, formación pedagógica, experiencia en investigación del cuerpo docente de la carrera y el $26,7 \%$ señala que el porcentaje es bajo

\section{DIMENSION: Ambiente Institucional}

\section{Cuadro 3}

\begin{tabular}{|l|l|l|l|l|l|}
\hline \multicolumn{2}{|c|}{} & Frecuencia & Porcentaje & $\begin{array}{l}\text { Porcentaje } \\
\text { válido }\end{array}$ & $\begin{array}{l}\text { Porcentaje } \\
\text { acumulado }\end{array}$ \\
\hline \multirow{3}{*}{ Válido } & Tiempo Completo & 10 & 66,7 & 66,7 & 66,7 \\
\cline { 2 - 6 } & Tiempo Posgrado & 5 & 33,3 & 33,3 & 100,0 \\
\cline { 2 - 6 } & Total & 15 & 100,0 & 100,0 & \\
\hline
\end{tabular}

\section{Figura 3}

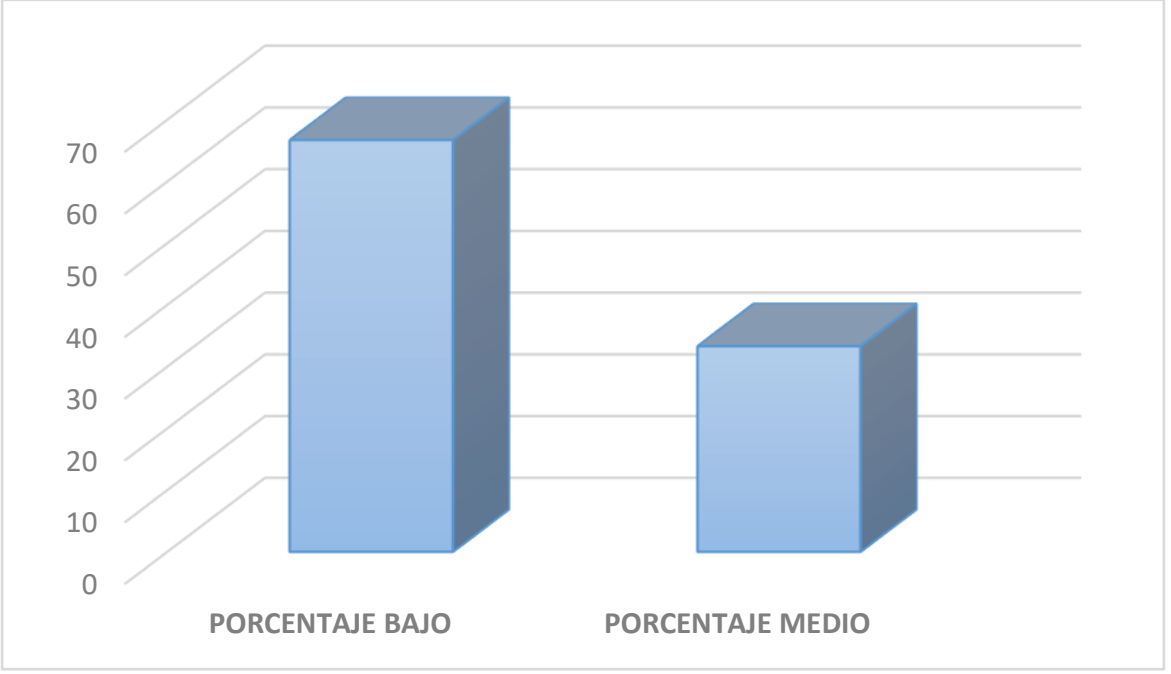

Interpretación: De acuerdo al cuadro estadístico y a la figura, se evidencia que el 66,7\% señala que tienen tiempo completo tiene un postgrado relevante para la carrera y el 33,3\% señala que tienen posgrado. 
Journal of the Academy $|114|$

Análisis descriptivo de la variable: Calidad del Desempeño Docente

Variable: Calidad del Desempeño Docente

Dimensión: Actividades De Docencia.

Cuadro 4

\begin{tabular}{|l|l|l|l|l|l|}
\hline \multicolumn{2}{|c|}{} & Frecuencia & Porcentaje & $\begin{array}{l}\text { Porcentaje } \\
\text { válido }\end{array}$ & $\begin{array}{l}\text { Porcentaje } \\
\text { acumulado }\end{array}$ \\
\hline \multirow{4}{*}{ Válido } & Nunca & 1 & 6,7 & 6,7 & 6,7 \\
\cline { 2 - 6 } & Casi Nunca & 3 & 20,0 & 20,0 & 26,7 \\
\cline { 2 - 6 } & A veces & 3 & 20,0 & 20,0 & 46,7 \\
\cline { 2 - 6 } & Casi Siempre & 2 & 13,3 & 13,3 & 60,0 \\
\cline { 2 - 6 } & Siempre & 6 & 40,0 & 40,0 & 100,0 \\
\cline { 2 - 6 } & Total & 15 & 100,0 & 100,0 & \\
\hline
\end{tabular}

Figura 4

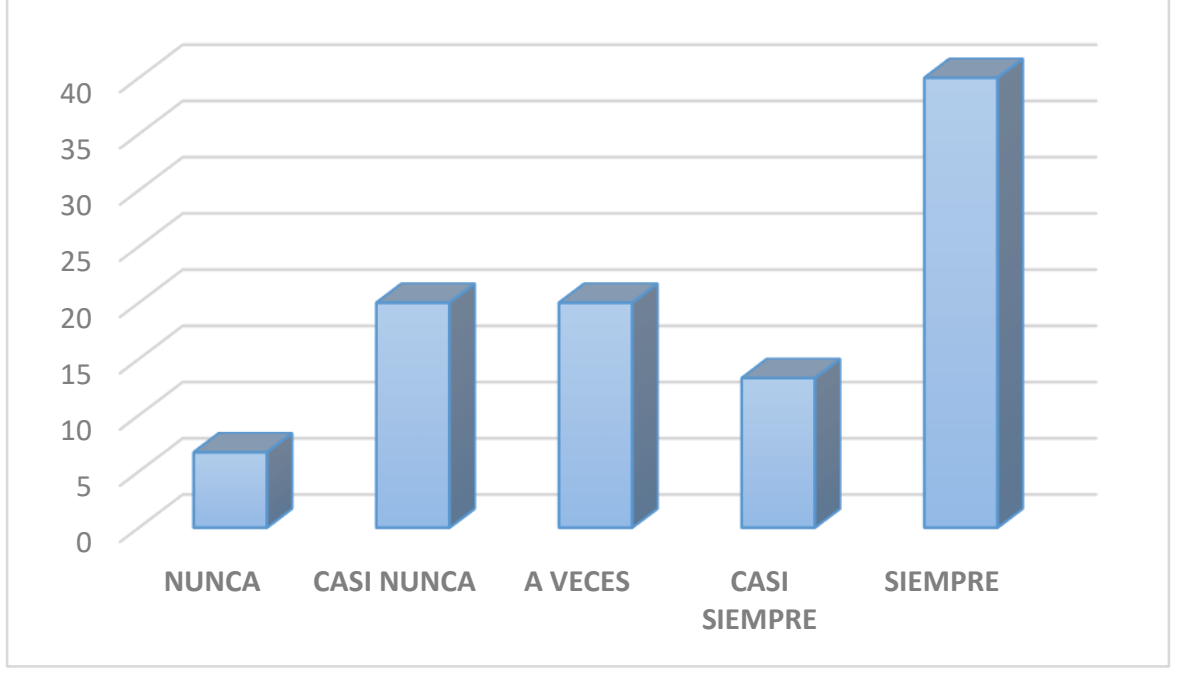

Interpretación: De acuerdo al cuadro estadístico y a la figura, se evidencia que el 40,0\% indica que siempre los docentes dominan los contenidos de la materia, asignatura o módulo, las competencias para el desempeño docente relacionadas con la sociabilidad pedagógica, con la gestión de clases (metodología), con el refuerzo de las habilidades y destrezas básicas de los estudiantes, con la motivación, y con los procedimientos de evaluación y medición de logros del aprendizaje, en la participación en el diseño macro curricular, en la asistencia regular al trabajo docente, en la participación en el diseño micro curricular y grado de su cumplimiento, en la elaboración de textos y otros materiales de apoyo didáctico, en la dirección de seminarios, organización de talleres y otros actos académicos, en la supervisión de las prácticas pre profesionales, en las traducciones de libros, artículos y notas, cuya incorporación en los programas de estudio sea necesaria o conveniente, en la dirección y lectura de tesis, disertaciones y trabajos de titulación o grado, en la participación en tribunales de titulación o grado, en las tutorías de trabajos de pregrado y postgrado el 20,0\% indica que a veces, el $20,0 \%$ indica que casi nunca, el 13,3\% indica que casi siempre y el 6,7\% indica que nunca. 
Journal of the Academy $|115|$

DIMENSION: Administración y Gestión Académica.

Cuadro 5

\begin{tabular}{|l|l|l|l|l|l|}
\hline \multicolumn{2}{|c|}{} & Frecuencia & Porcentaje & $\begin{array}{l}\text { Porcentaje } \\
\text { válido }\end{array}$ & $\begin{array}{l}\text { Porcentaje } \\
\text { acumulado }\end{array}$ \\
\hline \multirow{4}{*}{ Válido } & No aplica & 4 & 4,2 & 4,2 & 4,2 \\
\cline { 2 - 6 } & Ninguna & 26 & 27,4 & 27,4 & 31,6 \\
\cline { 2 - 6 } & Parcialmente & 25 & 26,3 & 26,3 & 57,9 \\
\cline { 2 - 6 } & En su mayor parte & 24 & 25,3 & 25,3 & 83,2 \\
\cline { 2 - 6 } & Totalmente & 16 & 16,8 & 16,8 & 100,0 \\
\cline { 2 - 6 } & Total & 95 & 100,0 & 100,0 & \\
\hline
\end{tabular}

\section{Figura 5}

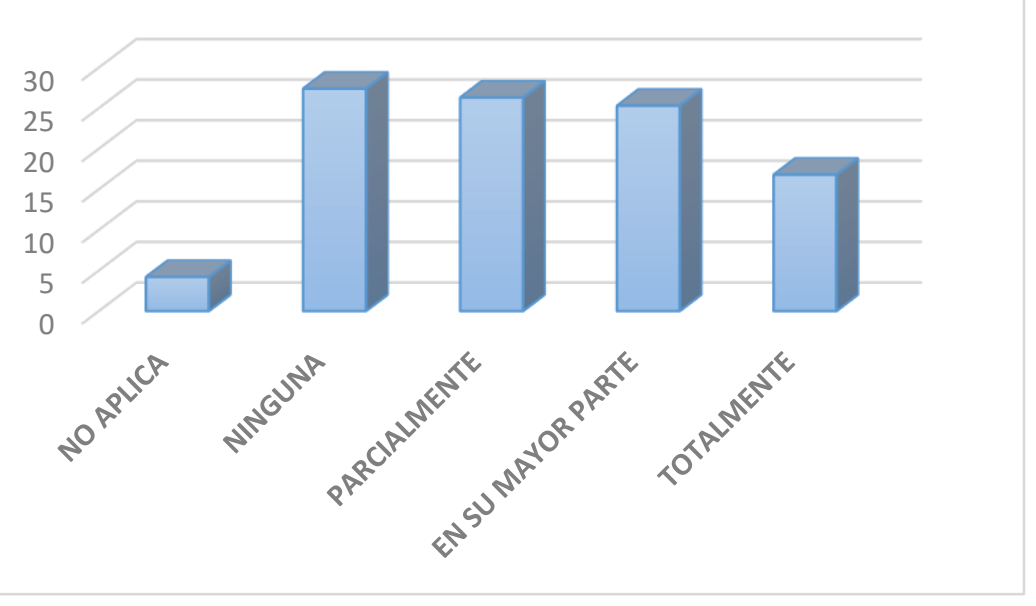

Interpretación: De acuerdo al cuadro estadístico y a la figura, se evidencia que el 27,4\% indica que ninguna en coordinación o dirección de: área, escuela, departamento, programa, facultad u otra forma de unidad académica, en la participación en comités o consejos académicos; en la participación en comisiones especiales, los docentes formulan y ejecutan de proyectos de investigación, difusión de resultados de la investigación, publicaciones en revistas especializadas y libros patentes e invenciones, obtención de becas, contribuciones y otras formas de ayuda para investigaciones y proyectos, organización y gestión de congresos, simposios, seminarios y otros actos académicos vinculados con la investigación, participación como invitado, con ponencias, comunicaciones o conferencias, en congresos académicos, simposios y seminarios, publicación de reseñas, investigaciones no publicadas, pasantías en instituciones académicas o empresas vinculadas a la actividad investigativa del profesor, el 26,3\% indica que parcialmente, el 25,3\% indica que, en su mayor parte, el 16,8\% indica que totalmente y el $4,2 \%$ indica que no aplica.

\section{DIMENSIÓN: Vinculación con la Colectividad.}

\section{Cuadro 6}

\begin{tabular}{|l|l|l|l|l|l|}
\hline \multicolumn{2}{|c|}{} & Frecuencia & Porcentaje & $\begin{array}{l}\text { Porcentaje } \\
\text { válido }\end{array}$ & $\begin{array}{l}\text { Porcentaje } \\
\text { acumulado }\end{array}$ \\
\hline \multirow{5}{*}{ Válido } & Nunca & 1 & 0,6 & 0,6 & 0,6 \\
\cline { 2 - 6 } & Casi nunca & 9 & 5,7 & 5,7 & 6,3 \\
\cline { 2 - 6 } & A veces & 55 & 34,8 & 34,8 & 41,1 \\
\cline { 2 - 6 } & Casi siempre & 57 & 36,1 & 36,1 & 77,2 \\
\cline { 2 - 6 } & Siempre & 36 & 22,8 & 22,8 & 100,0 \\
\cline { 2 - 6 } & Total & 158 & 100,0 & 100,0 & \\
\hline
\end{tabular}




\section{Journal of the Academy $|116|$}

\section{Figura 6}

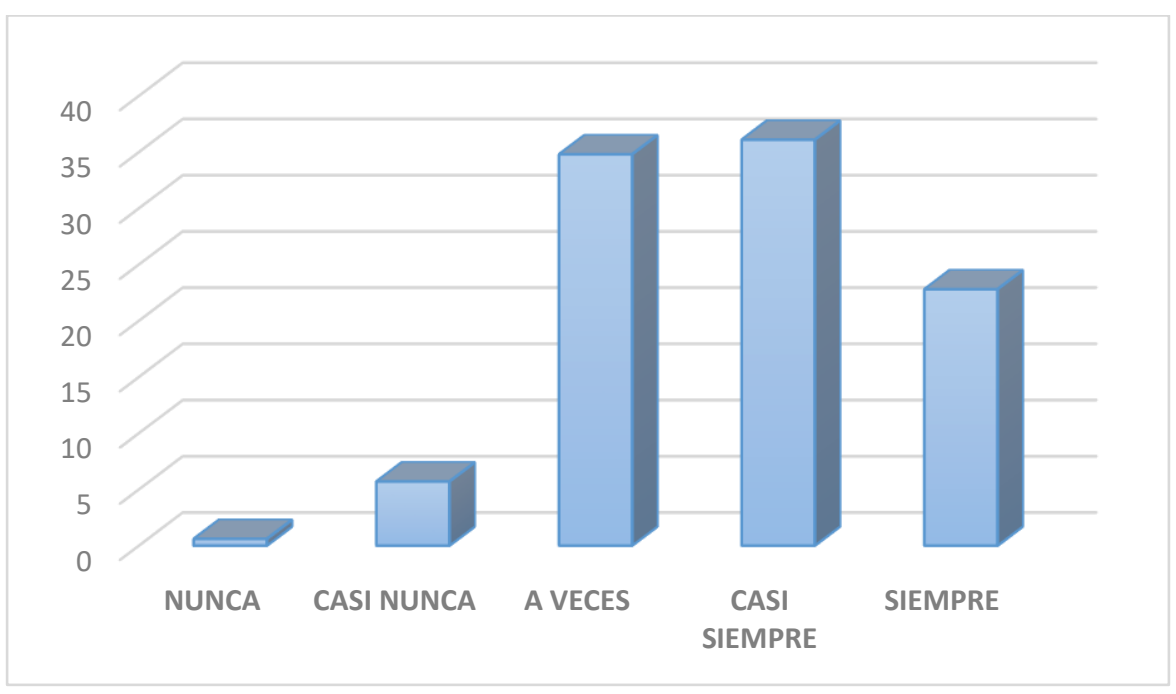

Interpretación: De acuerdo al cuadro estadístico y a la figura, se evidencia que el 36,1\% indica que casi siempre los docentes elaboran proyectos y negociaciones con empresas y organizaciones, dirección y participación en proyectos de capacitación, educación continua, consultorías y pasantías de profesores y estudiantes, dirección y participación en proyectos de extensión social o cultural de la institución, dirección y participación en proyectos de cooperación interuniversitaria, participación en organismos vinculados a la educación superior, la ciencia, la tecnología o la cultura, el 34,8\% indica que a veces, el $22,8 \%$ indica que siempre, el 5,7\% indica que casi nunca y el 0,6\% indica que nunca.

\section{Resultados}

Tras el análisis de los resultados de la investigación se obtiene que la correlación entre la acreditación de carreras profesional incide significativamente en la calidad del desempeño de los docentes universitarios, lo cual de acuerdo a la estadística corresponde a un nivel de correlación positiva. Asimismo, dentro de la evaluación de las otras dimensiones de la variable se ha comprobado que el plan curricular de las universidades influye significativamente en el desempeño de la calidad de enseñanza del docente universitario en el Ecuador. Lo que nos muestra un porcentaje del $100 \%$ que señala que existe una relación altamente significativa entre la calidad del docente universitario en el Ecuador y la necesidad de acreditar las carreras universitarias.

Lo mencionado anteriormente guarda relación con lo mencionado por Tuesta (2017), el cual considera que si se elabora un sistema operativo para ejecutar la autoevaluación de manera eficiente y efectiva, basado en un modelo teórico de gestión para la acreditación de la Escuela Profesional, que tenga en cuenta la relación entre la lógica de la formación de aseguramiento de la calidad y la lógica del proceso de auto evaluación y evaluación en el contexto formativo, que contribuye a potenciar una acreditación universitaria pertinente.

Asimismo, Cabarcas y Contreras (2019) establecen la relación entre la Evaluación de Desempeño Docente (EDD) y los componentes del Índice Sintético de Calidad Educativa (ISCE) cuyos resultados obtenidos describen en dos fases: una fase descriptiva y una fase inferencial en la que los hallazgos determinaron que los componentes desempeño, progreso y eficiencia alcanzaron una correlación positiva pero baja y estadísticamente no significativa.

Otro estudio sobre la acreditación fue desarrollado en la Universidad Mayor de Chile por Campo (2017) afirman que el aseguramiento de la calidad aplica dos modelos diferentes, pero buscando si es posible encontrar similitudes, diferentes, virtudes o aspectos que puedan servir de orientación en otras aplicaciones. Utilizó como estrategia la comparación, destacando elementos comunes y diferenciadores, 


\section{Journal of the Academy | $117 \mid$}

ventajas y desventajas, excluyentes y complementarios, de manera de interpretar y contextualizar, para emitir apreciaciones que den respuesta a las interrogantes.

Finalmente, Rubio (2003) señala que en el Programa de Mejoramiento del Profesorado ha propuesto líneas para la mejora de la calidad del docente universitario.

Estas estrategias son:

1. Reconocimiento a profesores con perfil deseable.

2. Apoyo a profesores con perfil deseable.

3. Apoyo a la reincorporación de ex-becarios PROMEP

4. Apoyo a la incorporación de nuevos profesores de tiempo completo.

5. Apoyo a la conexión de instituciones a Internet.

6. Registro de profesores de tiempo completo en cuerpos académicos.

7. Apoyo a la conformación de redes de cuerpos académicos.

8. Apoyo a la integración de nuevos cuerpos académicos.

9. Becas post doctorales en cuerpos académicos consolidados.

Lo señalado por el autor podría ser un marco para la consecución de nuevas alternativas que permita a los docentes.

\section{CONCLUSIONES}

Se concluye que la hipótesis de investigación es aceptada. Esto significa que existe una incidencia significativa entre la política de Evaluación y Acreditación de las carreras profesionales en la calidad del desempeño profesional de los docentes universitarios.

Los directivos y estudiantes perciben que la dimensión del plan curricular a través del Perfil de egreso, la Malla Curricular, Programas de las asignaturas, los Sílabos y la Practica Pre profesional incide significativamente con la calidad del desempeño profesional de los docentes a través del estado actual y prospectiva de la universidad.

Si existe incidencia significativa entre la dimensión del aspecto académico en la calidad del desempeño profesional de los docentes universitarios.

La dimensión del ambiente institucional incide significativamente en la calidad del desempeño profesional de los docentes universitarios. Los estudiantes inciden de manera significativa en la calidad del desempeño profesional de los docentes universitarios.

Asimismo, se recomienda considerar el informe que establece el Modelo de Evaluación Institucional de Universidades y Escuelas Politécnicas elaborado en el año 2015 por el CEAACES, porque en ella se exponen los elementos básicos que definen el proceso de evaluación universitario partiendo de la concepción que el mismo se enmarca en la búsqueda de la calidad de la educación superior.

Asimismo, es necesario que las autoridades universitarias consideren a la docencia universitaria como un rol fundamental para la transmisión del conocimiento a los estudiantes y por ende la urgencia de atenderla es necesaria, a través de diversos programas de capacitación especialización talleres, entre otros.

Todo lo mencionado con la finalidad de cumplir cabalmente el artículo 13, literal a), con respecto relativo a las funciones del sistema de educación superior en la que establece: "Garantizar el derecho a la educación superior mediante la docencia, la investigación y su vinculación con la sociedad, asegurando crecientes niveles de calidad, excelencia académica y pertinencia”. 


\section{Journal of the Academy $|118|$}

\section{REFERENCIAS BIBLIOGRÁFICAS}

Apunte, R. (2007). El proceso de evaluación y acreditación. funcionalidad y/o disfuncionalidad para las universidades del Ecuador. Tesis de Maestría. Universidad Andina Simón Bolívar. Ecuador.

Borroto, R., \& Salas, R. (2000). La acreditación y los créditos académicos como base de la calidad universitaria. Experiencia cubana. Educación Médica Superior, 14(1), 48-55. Recuperado de http://scielo.sld.cu/scielo.php?script=sci_arttext\&pid=S0864-21412000000100007\&lng=es\&tlng=pt.

Cabarcas, D. y Contreras, D. (2019). Evaluación del Desempeño Docente y su relación con las dimensiones del Índice Sintético de Calidad Educativa en instituciones públicas del Distrito de Barranquilla. Tesis de Maestría. Universidad de la Costa, Colombia: Barranquilla.

Campo, M. (2017). Estudio comparativo de dos modelos de acreditación universitaria: la aplicabilidad de estándares y criterios a la Universidad Mayor de Chile. Tesis de Doctorado. Universidad Nacional de Educación a Distancia. España: Madrid.

Consejo de Evaluación, Acreditación y Aseguramiento de la Calidad de la Educación Superior. (2013). "Categorización de universidades", Evaluación de universidades 2009, Recuperado de http://www.ceaaces.gob.ec/sitio/evaluacionuniversidades-

Guzmán, J. (2011). La calidad de la enseñanza en educación superior ¿Qué es una buena enseñanza en este nivel educativo? Perfiles educativos, 33(spe), 129-141. Recuperado de http://www.scielo.org.mx/scielo.php?script=sci arttext\&pid=S0185-

$\underline{26982011000500012 \& \operatorname{lng}=\mathrm{es} \& \ln g=\mathrm{es}}$

Hernández, R., Fernández, C., y Baptista, P. (2006). Metodología de la Investigación. Bogotá: McGraw Hill.

López, P. (2004). POBLACIÓN MUESTRA Y MUESTREO. Punto Cero, 09(08), 69-74. Recuperado de $\quad$ http://www.scielo.org.bo/scielo.php?script=sci_arttext\&pid=S181502762004000100012\&lng=es\&tlng=es.

Marum-Espinosa, E. (2003). Innovación en la docencia de pregrado: el reto de la calidad y la acreditación en México. Universidades, (26),23-36. Recuperado de https://www.redalyc.org/articulo.oa?id=373/37302604

República del Ecuador. (2010). Ley Orgánica de Educación Superior, recuperado de https://www.siteal.iiep.unesco.org/sites/default/files/sit accion files/ec 6011.pdf

Tuesta, E. (2017). La autoevaluación como parte del proceso de acreditación. Tesis de Doctorado. Universidad Señor De Sipán. Perú: Chiclayo. 